EUROPEAN JOURNAL OF PURE AND APPLIED MATHEMATICS

Vol. 15, No. 1, 2022, 144-157

ISSN 1307-5543 - ejpam.com

Published by New York Business Global

\title{
Periodic Solution of Caputo-Fabrizio Fractional Integro-differential Equation with Periodic and Integral Boundary Conditions
}

Ava Sh. Rafeeq

Department of Mathematics, Faculty of Science, University of Zakho, Duhok, Iraq

\begin{abstract}
In this paper, we study a new approach of investigation of existence, uniqueness and stability of the periodic solution of the nonlinear fractional integro-differential equation of type Caputo-Fabrizio fractional derivative with the initial condition, periodic boundary conditions, and integral boundary conditions by using successive approximations method and Banach fixed point theorem. Finally, some examples are present to illustrate the theorems.
\end{abstract}

2020 Mathematics Subject Classifications: 34A08, 26A33, 34G20, 34C25, 45J05

Key Words and Phrases: Caputo- Fabrizio fractional derivative, integro-differential equation, periodic and integral boundary conditions, Periodic solutions, successive approximation method, Banach fixed point theorem

\section{Introduction}

Fractional differential equations have been recognized in the last decade as important tools to describe the mathematical modeling of processes in the fields of physics, chemistry, engineering, statistics, aerodynamics, control theory, signal and image processing, etc. $[10,11,13]$. On the other hand, we observe periodic motions in every field of science and everywhere in real life [6]. The theory and applications of the fractional differential equations have recently been addressed by several researchers for a variety of problems, which we refer the reader to $[1,2,4]$. We mention here some of these definitions, such as Riemann-Liouville, Hadamard, Grünwald-Letnikov, Weyl, Riesz, Erdélyi-Kober, and Caputo. Compared with an integer order, a significant feature of a fractional order differential operator appeared in its hereditary property. In other words, when we describe a process by a fractional operator, we predict the future state by its current as well as its past states $[14,16]$.

However, the new definition suggested by Caputo and Fabrizio [5], which has all the characteristics of the old definitions, assumes two different representations for the temporal and spatial variables. They claimed that the classical definition given by Caputo

DOI: https://doi.org/10.29020/nybg.ejpam.v15i1.4247

Email address: ava.rafeeq@uoz.edu.krd (A. S. Rafeeq) 
appears to be particularly convenient for mechanical phenomena, related to plasticity, fatigue, damage, and with electromagnetic hysteresis. The main advantage of the CaputoFabrizio approach is that the boundary conditions of the fractional differential equations with Caputo-Fabrizio derivatives admit the same form as for the integer-order differential equations. On the other hand, the Caputo-Fabrizio fractional derivative has many significant properties, such as its ability in describing matter heterogeneities and configurations with different scales $[12,20]$.

In [21], we have the analytic solutions of a viscous fluid with the Caputo and CaputoFabrizio fractional derivatives. In [8], the authors used the fractional derivative with a nonsingular kernel to model a Maxwell fluid and found semianalytical solutions. In [22], we found a comparison approach of two latest fractional derivatives models, namely, Atangana-Baleanu and Caputo- Fabrizio, for a generalized Casson fluid and obtained exact solutions. Due to the abovementioned applications, the existence of solutions for nonlinear differential equations is an attractive research topic and has been studied using different techniques of nonlinear analysis $[9,18]$. One of the most important theorems in ordinary differential equations is Picard's existence and uniqueness theorem. This theorem, which is applied on first-order ordinary differential equations, can be generalized to establish existence and uniqueness results for both higher-order ordinary differential equations and systems of differential equations $[3,7,15,17]$.

In this paper, we investigate the existence and approximate periodic solution of the following nonlinear fractional integro-differential equation:

$$
{ }_{0}^{C F} D_{t}^{\alpha}(u(t))=h\left(t, u(t), \int_{0}^{a(t)} \mathrm{g}(\mathrm{s}, u(s)) d s\right)
$$

such that $t \in J=[0, T]$, with the initial condition $\mathrm{u}(0)=\mathrm{u}_{0}$, where ${ }_{0}^{\mathrm{CF}} D_{t}^{\alpha}$ denotes the Caputo-Fabrizio fractional derivative $(\alpha \in(0,1])$. We extend Picard's theorem to this problem, and by the successive approximation method, an iterative process is provided to obtain the periodic solution.

\section{Preliminaries}

In this section, we recall some notations and definitions which are needed throughout this paper. Further, some lemmas and theorems are stated as preparations for the main results. First, in the following, we provide some basic concepts and definitions in connection with the new Caputo-Fabrizio derivative.

Let $H^{1}(a, b)=\left\{g \mid g \in L^{2}(a, b), g^{\prime} \in L^{2}(a, b)\right\}$, where $L^{2}(a, b)$ is the space of square integrable functions on the interval $(a, b)$.

Definition 1. [10] For a function $g:(0, \infty) \rightarrow \mathbb{R}$, the Caputo derivative of order $\alpha>0$ of $g$ is defined by

$$
{ }_{0}^{t} D^{\alpha} g(t)=\frac{1}{\Gamma(n-\alpha)} \int_{0}^{t}(t-s)^{n-\alpha-1} g^{(n)}(s) d s
$$


where $n=[\alpha]+1$ and $[\alpha]$ denotes the integer part of $\alpha$, and $\Gamma($.$) denotes the Gamma$ function, i.e., $\Gamma(z)=\int_{0}^{\infty} e^{-t} t^{z-1} d t$

Definition 2. [10] Let $g$ be a function which is defined almost everywhere a.e on $[a, b]$, for $\alpha>0$, we define

$$
{ }_{a}^{b} D^{-\alpha} f=\frac{1}{\Gamma(\alpha)} \int_{a}^{b}(b-t)^{a-1} g(t) d t
$$

provided that the integral (Lebesgue)exists.

Definition 3. [5] Let $g$ be a given function in $H^{1}(a, b)$. The Caputo-Fabrizio derivative of fractional order $\alpha \in(0,1)$ is defined as

$$
{ }_{a}^{C F} D_{\mathrm{t}}^{\alpha}(g(t))=\left(\frac{N(\alpha)}{1-\alpha}\right) \int_{a}^{t} g^{\prime}(x) \exp \left[-\alpha \frac{t-x}{1-\alpha}\right] d x
$$

where $N(\alpha)$ is a normalization function. Also, if a certain function $g$ does not satisfy in the restriction $g \in H^{1}(a, b)$, then its fractional derivative is redefined as

$$
{ }_{a}^{C F} D_{t}^{\alpha}(g(t))=\frac{\alpha N(\alpha)}{1-\alpha} \int_{a}^{t}(g(t)-g(x)) \exp \left[-\alpha \frac{t-x}{1-\alpha}\right] d x
$$

Clearly, if one sets $\sigma=(1-\alpha) / \alpha \in(0, \infty)$ and $\alpha=1 /(1+\sigma) \in(0,1)$, then the CaputoFabrizio definition becomes

$$
{ }_{a}^{C F} D_{t}^{e}(g(t))=\frac{N(\sigma)}{\sigma} \int_{a}^{t} g^{\prime}(x) \exp \left[-\frac{t-x}{\sigma}\right] d x
$$

where $N(0)=N(\infty)=1$, and

$$
\lim _{\sigma \rightarrow 0} \exp \left[-\frac{t-x}{\sigma}\right]=\delta(x-t)
$$

Also, the fractional derivative of order $(n+\alpha)$ when $n \geq 1$ and $\alpha \in[0,1]$ is defined by the following

$$
{ }_{a}^{C F} D_{t}^{(a+n)}(g(t))=\alpha^{C F} D_{t}^{(a)}\left(D_{t}^{(n)} g(t)\right)
$$

Definition 4. [5] Let $g \in H^{1}(a, b)$, then its fractional integral of an arbitrary order is defined as follows:

$$
{ }_{\alpha} C_{t}^{x}(g(t))=\frac{2(1-\alpha)}{(2-\alpha) N(\alpha)} g(t)+\frac{2 \alpha}{(2-\alpha) N(\alpha)} \int_{a}^{t} g(s) d s, t \geq 0
$$

It is dear, in view of the abowe definition, that the $\alpha$ th Caputo-Fabrizio derivative of function $g$ is average between $g$ and its first-order integral. Therefore,

$$
\frac{2(1-\alpha)}{(2-\alpha) N(\alpha)}+\frac{2 \alpha}{(2-\alpha) N(\alpha)}=1
$$

So, we arrive at the following

$$
N(\alpha)=\frac{2}{2-\alpha}, 0 \leq \alpha \leq 1
$$


Definition 5. The periodic solution of the fractional integro-differential equation (1.1), with initial condition $u(0)=u_{0}$ and periodic boundary condition $u(0)=u(T)$ are defining the following integral equation

$$
\begin{aligned}
& u\left(t, u_{0}\right)=\mathrm{u}_{0}+\frac{2(1-\alpha)}{(2-\alpha) N(\alpha)} h\left(t, u(t), \int_{0}^{a(t)} \mathrm{g}(\mathrm{s}, u(s)) d s\right) \\
& -\left(\frac{2(1-\alpha)}{(2-\alpha) N(\alpha)} \frac{1}{\mathrm{~T}} \int_{0}^{T} h\left(s, u(s), \int_{0}^{a(s)} \mathrm{g}(\tau, u(\tau)) d \tau\right) d s+\frac{2 \alpha}{(2-\alpha) N(\alpha)}\right. \\
& \int_{0}^{t}\left(h\left(s, u(s), \int_{0}^{a(s)} \mathrm{g}(\tau, u(\tau)) d \tau\right)-\frac{1}{\mathrm{~T}} \int_{0}^{T} h\left(s, u(s), \int_{0}^{a(s)} \mathrm{g}(\tau, u(\tau)) d \tau\right) d s\right) d s
\end{aligned}
$$

For all $t \in J$.

Lemma 1. [19] Let $g(t)$ be a vector function which is defined in the interval $0 \leq t \leq T$, then:

$$
\left|\int_{0}^{t}\left(g(s)-\frac{1}{T} \int_{0}^{T} g(s) d s\right) d s\right| \leq \beta(t) M
$$

where $M=\max _{t \in[0, T]}|g(t)|$ and $\beta(t)=2 t\left(1-\frac{t}{T}\right), \max _{t \in[0, T]}|\beta(t)| \leq \frac{T}{2}$.

The proof follows directly from the estimate:

$$
\begin{aligned}
\left|\int_{0}^{t}\left(g(s)-\frac{1}{T} \int_{0}^{T} g(s) d s\right) d s\right| & \leq\left(1-\frac{t}{T}\right) \int_{0}^{t}|g(s)| d s+\frac{t}{T} \int_{t}^{T}|g(s)| d s \\
& \leq \beta(t) M
\end{aligned}
$$

Theorem 1. [10] (Banach fixed point theorem). Let $(E,\|\|$.$) be a Banach space and$ $P: E \rightarrow E$ be a contraction mapping i.e. Lipchitz continuous with Lipchitz constant $L \in[0,1)$. Then $\varphi \in E$ has a unique fixed point.

\section{Conditions for Convergence of Successive Approximation}

Some conditions are needed for investigate of the successive approximation for periodic solution of the problem (1.1) with $\mathrm{u}(0)=\mathrm{u}_{0}$, suppose that the functions $h \in$ $C\left([0, T] \times D_{1} \times D_{2}, \mathbb{R}\right), g \in C\left([0, T] \times D_{1}, \mathbb{R}\right), D_{1}$ and $D_{2}$ are compact subset of $\mathbb{R}, a(t)$ is continuous functions on $[0, T]$, moreover define $||=.\max _{t \in[0, T]}|$.$| , and satisfies the follow-$ ing hypothesis.

$\mathrm{H}_{1}$ There exist positive constants $M, L, k_{1}, k_{2}$, and $L_{1}$, such that

$$
\begin{gathered}
|h(t, u, z)| \leq M \quad(3.1) \\
|g(t, u)| \leq L \quad(3.2) \\
\left|h\left(t, u_{1}, z_{1}\right)-h\left(t, u_{2}, z_{2}\right)\right| \leq k_{1}\left|u_{1}-u_{2}\right|+k_{2}\left|z_{1}-z_{2}\right| \\
\left|g\left(t, u_{1}\right)-g\left(t, u_{2}\right)\right| \leq L_{1}\left|u_{1}-u_{2}\right|
\end{gathered}
$$

where $z_{i}=\int_{0}^{a(t)} \mathrm{g}\left(\mathrm{s}, u_{i}(s)\right) d s$ and for all $t \in[0, T], \quad u, u_{1}, u_{2} \in D_{1}$ and $z_{i} \in D_{2}, \quad i=1,2$ $\mathrm{H}_{2}$ : There exist positive constants $a_{T}$, such that for $t \in[0, T]$,

$$
|a(t)| \leq a_{T}
$$


Define the non-empty set

$$
\mathrm{D}_{\mathrm{h}}=\mathrm{D}_{1}-M_{1}
$$

where

$$
M_{1}=\left(2(1-\alpha)+\frac{\alpha T}{2}\right) M
$$

Furthermore, we suppose that the following condition is valid:

$$
\Lambda=\left(2(1-\alpha)+\frac{\alpha T}{2}\right)\left(k_{1}+a_{T} L_{1} k_{2}\right)<1
$$

\section{Main Results}

Our main results separate to the following parts:

\subsection{Approximation of Periodic Solution of (1.1)}

In this section, we study the periodic approximation solutions of nonlinear fractional integro-differential equations $(1.1)$ with $\mathrm{u}(0)=\mathrm{u}_{0}$. In the beginning, we define the following sequence of functions $\left\{u_{m+1}\right\}_{m=0}^{\infty}$ given by the iterative formulas

$$
\begin{aligned}
& u_{m+1}\left(t, u_{0}\right)=u_{0}+\frac{2(1-\alpha)}{(2-\alpha) N(\alpha)} h\left(t, u_{m}(t), \int_{0}^{a(t)} g\left(s, u_{m}(s)\right) d s\right) \\
& -\frac{2(1-\alpha)}{(2-\alpha) N(\alpha)} \frac{1}{T} \int_{0}^{T} h\left(s, u_{m}(s), \int_{0}^{a(s)} g\left(\tau, u_{m}(\tau)\right) d \tau\right) d s+\frac{2 \alpha}{(2-\alpha) N(\alpha)} \\
& \int_{0}^{t}\left(h\left(s, u_{m}(s), \int_{0}^{a(s)} \mathrm{g}\left(\tau, u_{m}(\tau)\right) d \tau\right)-\frac{1}{\mathrm{~T}} \int_{0}^{T} h\left(s, u_{m}(s), \int_{0}^{a(s)} \mathrm{g}\left(\tau, u_{m}(\tau)\right) d \tau\right) d s\right) d s
\end{aligned}
$$

For all $t \in J, u_{0}(t)=u_{0}, m=0,1,2, \ldots$,

then will be introduced by the following theorems.

Theorem 2. If the nonlinear fractional integro-differential equation (1.1) with $\mathrm{u}(0)=\mathrm{u}_{0}$ satisfy the conditions $H_{1}$, and $H_{2}$, then the sequence of functions (4.1), which are periodic in $t$ of period $T$, converges uniformly as $m \rightarrow \infty$ on the domain:-

$$
\left(\mathrm{t}, \mathrm{u}_{0}\right) \in[0, T] \times D_{1}
$$

to the limit functions $\mathrm{u}_{\theta}$ defined on the domain (4.2) which is periodic in $\mathrm{t}$ of period $\mathrm{T}$ and satisfies the following integral equations:

$$
\begin{aligned}
& u\left(t, u_{0}\right)=\mathrm{u}_{0}+\frac{2(1-\alpha)}{(2-\alpha) N(\alpha)} h\left(t, u(t), \int_{0}^{a(t)} \mathrm{g}(\mathrm{s}, u(s)) d s\right) \\
& -\left(\frac{2(1-\alpha)}{(2-\alpha) N(\alpha)} \frac{1}{\mathrm{~T}} \int_{0}^{T} h\left(s, u(s), \int_{0}^{a(s)} \mathrm{g}(\tau, u(\tau)) d \tau\right) d s+\frac{2 \alpha}{(2-\alpha) N(\alpha)}\right. \\
& \int_{0}^{t}\left(h\left(s, u(s), \int_{0}^{a(s)} \mathrm{g}(\tau, u(\tau)) d \tau\right)-\frac{1}{\mathrm{~T}} \int_{0}^{T} h\left(s, u(s), \int_{0}^{a(s)} \mathrm{g}(\tau, u(\tau)) d \tau\right) d s\right) d s
\end{aligned}
$$

on the domain (4.2), provided that

$$
\left|u\left(t, u_{0}\right)-u_{m+1}\left(t, u_{0}\right)\right| \leq \Lambda^{\mathrm{m}}(\mathrm{E}-\Lambda)^{-1} \mathrm{M}_{1}
$$


for all $\mathrm{m} \geq 0, \mathrm{u}_{0} \in \mathrm{D}$, and $\mathrm{t} \in \mathrm{J}$

Proof. Setting $\mathrm{m}=0$ in the sequence of functions (4.1) and by using Lemma 1, we have

$$
\begin{aligned}
\left|u_{1}\left(t, u_{0}\right)-u_{0}\right| & \leq\left(\frac{4(1-\alpha)}{(2-\alpha) N(\alpha)}+\frac{2 \alpha}{(2-\alpha) N(\alpha)} \beta(t)\right) M \\
& \leq\left(2(1-\alpha)+\frac{\alpha T}{2}\right) M=M_{1}
\end{aligned}
$$

for all $t \in[0, T], u_{0} \in D_{h}$ we get $u_{1}\left(t, u_{0}\right) \in D_{1}$. Thus by mathematical induction, we find that

$$
\left|u_{m}\left(t, u_{0}\right)-u_{0}\right| \leq \mathrm{M}_{1}
$$

mean that for all $t \in[0, T], u_{0} \in D_{h}$ we get $u_{m}\left(t, u_{0}\right) \in D_{1}, m=0,1,2, \ldots$

Now, we claim that the sequences of functions (4.1) are uniformly convergent on the domain (4.2). By the inequalities (3.3)-(3.5), we obtain

$$
\begin{aligned}
\left|u_{m+1}\left(t, u_{0}\right)-u_{m}\left(t, u_{0}\right)\right| & \leq\left(2(1-\alpha)+\frac{\alpha T}{2}\right)\left(k_{1}+a_{T} L_{1} k_{2}\right) \\
& \left|u_{m}\left(t,, u_{0}\right)-u_{m-1}\left(t,, u_{0}\right)\right| \\
& =\Lambda\left|u_{m}\left(t, u_{0}\right)-u_{m-1}\left(t_{r}, u_{0}\right)\right|
\end{aligned}
$$

By mathematical induction, we obtain that

$$
\left|u_{m+1}\left(t, \mathrm{u}_{0}\right)-u_{m}\left(t, \mathrm{u}_{0}\right)\right| \leq \Lambda^{\mathrm{m}}\left|u_{1}\left(t, \mathrm{u}_{0}\right)-\mathrm{u}_{0}\right|
$$

Now from $\mathrm{m}=1,2, \ldots$ and $\mathrm{p} \geq 1$, we find that

$$
\begin{aligned}
\left|u_{m+p}\left(t, \mathrm{u}_{0}\right)-u_{m}\left(t, \mathrm{u}_{0}\right)\right| & \leq \Lambda^{\mathrm{m}}(1-\Lambda)^{-1}\left(\left(2(1-\alpha)+\frac{\alpha T}{2}\right) M\right) \\
& \leq \Lambda^{\mathrm{m}}(1-\Lambda)^{-1} M_{1},
\end{aligned}
$$

for all $\mathrm{t} \in[0, \mathrm{~T}], u_{0} \in \mathrm{D}_{\mathrm{h}}$.

Since $\Lambda=\left(2(1-\alpha)+\frac{\alpha T}{2}\right)\left(k_{1}+a_{T} L_{1} k_{2}\right)<1$ and $\lim _{m \rightarrow \infty} \Lambda^{\mathrm{m}}=0$, so that the right side of (4.8) tends to zero. Therefore the sequence of functions $u_{m}\left(t, u_{0}\right), m=1,2,3, \ldots$ is converges uniformly on the domain (4.2) to the limit function $\mathrm{u}\left(\mathrm{t}, \mathrm{u}_{0}\right)$ which is defined on the same domain. Let

$$
\lim _{m \rightarrow \infty} u_{m}\left(t, u_{0}\right)=\mathrm{u}_{\theta}\left(t, u_{0}\right)
$$

Since the sequence of functions (4.1) are periodic in $t$ of period $\mathrm{T}$, then the limiting function $\mathrm{u}_{\theta}\left(\mathrm{t}, \mathrm{u}_{0}\right)$ is also periodic in $\mathrm{t}$ of period T. By using the relation (4.9) and proceeding in (4.1) to limit, when $\mathrm{m} \rightarrow \infty$, it is converging that the limiting function $u\left(t, u_{0}\right)$ is the periodic solution of the integral equation (4.3).

Theorem 3. If all assumptions of the Theorem 2 are satisfy, then $\mathrm{u}\left(\mathrm{t}, \mathrm{u}_{0}\right)$ is a unique solution of the problem ( 1.1 ) with $\mathrm{u}(0)=\mathrm{u}_{0}$. 
Proof. Assume that $\hat{u}\left(t, u_{0}\right)$ is another solution of the problem (1.1) with $u(0)=u_{0}$, as follows

$\hat{u}\left(t, u_{0}\right)=\mathrm{u}_{0}+\frac{2(1-\alpha)}{(2-\alpha) N(\alpha)} h\left(t, \hat{u}(t), \int_{0}^{a(t)} \mathrm{g}(\mathrm{s}, \hat{u}(s)) d s\right)$
$-\left(\frac{2(1-\alpha)}{(2-\alpha) N(\alpha)} \frac{1}{\mathrm{~T}} \int_{0}^{T} h\left(s, \hat{u}(s), \int_{0}^{a(s)} \mathrm{g}(\tau, \hat{u}(\tau)) d \tau\right) d s+\frac{2 \alpha}{(2-\alpha) N(\alpha)}\right.$
$\int_{0}^{t}\left(h\left(s, \hat{u}(s), \int_{0}^{a(s)} \mathrm{g}(\tau, \hat{u}(\tau)) d \tau\right)-\frac{1}{\mathrm{~T}} \int_{0}^{T} h\left(s, \hat{u}(s), \int_{0}^{a(s)} \mathrm{g}(\tau, \hat{u}(\tau)) d \tau\right) d s\right) d s$

Now, the difference between the two solutions $\mathrm{u}\left(\mathrm{t}, \mathrm{u}_{0}\right)$ and $\hat{\mathrm{u}}\left(\mathrm{t}, \mathrm{u}_{0}\right)$,for all $\mathrm{t} \in[0, \mathrm{~T}]$ and $\mathrm{u}_{0} \in \mathrm{D}_{\mathrm{h}}$, hence, by the inequalities $(3,3)-(3.5)$, we get

$$
\begin{aligned}
\left|\mathrm{u}\left(\mathrm{t}, \mathrm{u}_{0}\right)-\hat{\mathrm{u}}\left(\mathrm{t}, \mathrm{u}_{0}\right)\right| & \leq\left(2(1-\alpha)+\frac{\alpha T}{2}\right)\left(k_{1}+a_{Y} L_{1} k_{2}\right)\left|\mathrm{u}\left(\mathrm{t}, \mathrm{u}_{0}\right)-\hat{\mathrm{u}}\left(\mathrm{t}, \mathrm{u}_{0}\right)\right| \\
& \leq \Lambda\left|\mathrm{u}\left(\mathrm{t}_{r} \mathrm{u}_{0}\right)-\hat{\mathrm{u}}\left(\mathrm{t}, \mathrm{u}_{0}\right)\right|
\end{aligned}
$$

By mathematical induction, we find that

$$
\left|\mathrm{u}\left(\mathrm{t}, \mathrm{u}_{0}\right)-\hat{\mathrm{u}}\left(\mathrm{t}, \mathrm{u}_{0}\right)\right| \leq \Lambda^{\mathrm{m}}\left|\mathrm{u}\left(\mathrm{t}, \mathrm{u}_{0}\right)-\hat{\mathrm{u}}\left(\mathrm{t}, \mathrm{u}_{0}\right)\right|
$$

From the condition $(3.7)$, shows that the solution $u\left(t, u_{0}\right)=\hat{u}\left(t, u_{0}\right)$, thus $u\left(t, u_{0}\right)$ is a unique periodic solution on the domain (4.2).

\subsection{Existence of Periodic Solutions of (1.1)}

The problem of the existence of the periodic solution for the problem (1.1) with $\mathrm{u}(0)=$ $\mathrm{u}_{0}$ is uniquely connected with the existence of the zeros of the functions:-

$$
\mu\left(0, \mathrm{u}_{0}\right)=\frac{1}{\mathrm{~T}} \int_{0}^{T} h\left(s, u(s), \int_{0}^{a(s)} \mathrm{g}(\tau, u(\tau)) d \tau\right) d s
$$

Also, we define the sequences of functions $\mu_{m}\left(0, u_{0}\right)$ are approximately determined by the following:

$$
\mu_{\mathrm{m}}\left(0, \mathrm{u}_{0}\right)=\frac{1}{\mathrm{~T}} \int_{0}^{T} h\left(s, u_{m}(s), \int_{0}^{a(s)} \mathrm{g}\left(\tau, u_{m}(\tau)\right) d \tau\right) d s
$$

Theorem 4. If the hypotheses and all the conditions of the theorem 2 are given, the following inequalities are satisfied:-

$$
\left|\mu\left(0, \mathrm{u}_{0}\right)-\mu_{\mathrm{m}}\left(0, \mathrm{u}_{0}\right)\right| \leq\left(k_{1}+a_{T} L_{1} k_{2}\right) \Lambda^{\mathrm{m}}(1-\Lambda)^{-1} M_{1}
$$

holds for all $\mathrm{m} \geq 0$

Proof. From equations (4.13) to (4.14), we obtain that

$$
\begin{aligned}
\left|\mu\left(0, \mathrm{u}_{0}\right)-\mu_{m}\left(0, \mathrm{u}_{0}\right)\right| & \leq\left(k_{1}+a_{T} L_{1} k_{2}\right)\left|u\left(t, \mathrm{u}_{0}\right)-u_{m}\left(t, \mathrm{u}_{0}\right)\right| \\
& \leq\left(k_{1}+a_{r} L_{1} k_{2}\right) \Lambda^{\mathrm{m}}(1-\Lambda)^{-1} M_{1}
\end{aligned}
$$


The inequality (4.15) is hold for all $\mathrm{m} \geq 0$.

Theorem 5. Let the function $h(s, u(s), z(t))$ be defined on the intervals $[c, d]$ on $R$ and periodic in $t$ of period $T$, suppose that for all $\mathrm{m} \geq 0$, then the sequences of the functions $\mu_{\mathrm{m}}\left(0, u_{0}\right)$ which are defined in (4.14) satisfy the inequalities:-

$$
\left.\begin{array}{l}
\min _{u_{0} \in[c, d]} \mu_{\mathrm{m}}\left(0, \mathrm{u}_{0}\right) \leq-\left(k_{1}+a_{\tau} L_{1} k_{2}\right) \Lambda^{\mathrm{m}}(1-\Lambda)^{-1} M_{1} \\
\max _{u_{0} \in[c, d]} \mu_{\mathrm{m}}\left(0, \mathrm{u}_{0}\right) \geq\left(k_{1}+a_{r} L_{1} k_{2}\right) \Lambda^{\mathrm{m}}(1-\Lambda)^{-1} M_{1}
\end{array}\right\}
$$

Then the problem (1.1) has a periodic solution $\mathrm{u}\left(\mathrm{t}, \mathrm{u}_{0}\right)$ such that $\mathrm{u}_{0} \in[\mathrm{c}, \mathrm{d}]=\left[\mathrm{c}+M_{1}, \mathrm{~d}-M_{1}\right]$

Proof. Let $u_{1}$ and $u_{2}$ be any points belonging to the intervals $[c, d]$, such that

$$
\left.\begin{array}{l}
\mu_{\mathrm{m}}\left(0, \mathrm{u}_{1}\right)=\min _{u_{0} \in[c, d]} \mu_{\mathrm{m}}\left(0, \mathrm{u}_{0}\right) \\
\mu_{\mathrm{m}}\left(0, \mathrm{u}_{2}\right)=\max _{u_{0} \in[c, d]} \mu_{\mathrm{m}}\left(0, \mathrm{u}_{0}\right)
\end{array}\right\}
$$

By using inequalities (4.15) to (4.18),, the following are obtained:-

$$
\left.\begin{array}{l}
\mu\left(0, \mathrm{u}_{1}\right)=\mu_{\mathrm{m}}\left(0, \mathrm{u}_{1}\right)+\left(\mu\left(0, \mathrm{u}_{1}\right)-\mu_{\mathrm{m}}\left(0, \mathrm{u}_{1}\right)\right)<0 \\
\mu\left(0, \mathrm{u}_{2}\right)=\mu_{\mathrm{m}}\left(0, \mathrm{u}_{2}\right)+\left(\mu\left(0, \mathrm{u}_{2}\right)-\mu_{m}\left(0, \mathrm{u}_{2}\right)\right)>0
\end{array}\right\}
$$

and from the continuity of the functions $\mu\left(0, u_{1}\right), \mu\left(0, u_{2}\right)$ and the inequalities (4.19), then the isolated singular points $u^{0} \in[c, d]$ exist such that $\mu\left(0, u^{0}\right)=0$. This means that (1.1) has a periodic solution $\mathrm{u}\left(\mathrm{t}, \mathrm{u}_{0}\right)$.

\subsection{Stability of Periodic Solution of (1.1)}

In this section, we investigate the stability or periodic solution of (1.1).

Theorem 6. Let the function $\mu\left(0, \mathrm{u}_{0}\right)$ be defined by the equation (4.13) where $\mathrm{u}\left(\mathrm{t}, \mathrm{u}_{0}\right)$ is a limit of the sequence of the function (4.1), then the following inequalities yield:-

$$
\left|\mu\left(0, u_{0}\right)\right| \leq M
$$

and

$$
\left|\mu\left(0, \mathrm{u}_{0}^{1}\right)-\mu\left(0, \mathrm{u}_{0}^{2}\right)\right| \leq F_{2} F_{3}\left|u_{0}^{1}-u_{0}^{2}\right|
$$

where

$$
F_{1}=2(1-\alpha)+\frac{\alpha T}{2}, \quad F_{2}=k_{1}+a_{T} L_{1} k_{2}, \quad F_{3}=\left(1-F_{1} F_{2}\right)^{-1}
$$

Proof From the properties of the function $u\left(t, u_{0}\right)$ as in the Theorem 2, the function $\mu\left(0, \mathrm{u}_{0}\right), u_{0} \in D$ is continuous and bounded by $\frac{1-\alpha}{\alpha T} g^{T}+M$ in the domain (4.2). From (4.13), we obtained that

$$
\left|\mu\left(0, \mathrm{u}_{0}\right)\right| \leq \frac{1}{\mathrm{~T}} \int_{0}^{T}\left|h\left(s, u(s), \int_{0}^{a(s)} \mathrm{g}(\tau, u(\tau)) d \tau\right)\right| d s \leq M
$$


Next, from inequality (4.13), we get

$$
\begin{aligned}
\mid \mu\left(0, \mathrm{u}_{0}^{1}\right) & -\mu\left(0, \mathrm{u}_{0}^{2}\right)\left|\leq\left(k_{1}+a_{T} L_{1} k_{2}\right)\right| u\left(t, u_{0}^{1}\right)-u\left(t, u_{0}^{2}\right) \mid \\
& \leq F_{2}\left|u\left(t, u_{0}^{1}\right)-u\left(t, u_{0}^{2}\right)\right|
\end{aligned}
$$

where the functions $\mathrm{u}\left(\mathrm{t}, \mathrm{u}_{0}^{1}\right)$ and $\mathrm{u}\left(\mathrm{t}, u_{0}^{2}\right)$ are solutions of the integral equation:-

$$
\begin{aligned}
& u\left(t, \mathrm{u}_{0}^{\mathrm{k}}\right)=\mathrm{u}_{0}^{\mathrm{k}}+\frac{2(1-\alpha)}{(2-\alpha) N(\alpha)} h\left(t, u\left(t, \mathrm{u}_{0}^{\mathrm{k}}\right), \int_{0}^{a(t)} \mathrm{g}\left(\mathrm{s}, u\left(s, \mathrm{u}_{0}^{\mathrm{k}}\right)\right) d s\right)- \\
& \frac{2(1-\alpha)}{(2-\alpha) N(\alpha)} \frac{1}{\mathrm{~T}} \int_{0}^{T} h\left(s, u\left(s, \mathrm{u}_{0}^{\mathrm{k}}\right), \int_{0}^{a(s)} \mathrm{g}\left(\tau, u\left(\tau, \mathrm{u}_{0}^{\mathrm{k}}\right)\right) d \tau\right) d s+ \\
& \frac{2 \alpha}{(2-\alpha) N(\alpha)} \int_{0}^{t}\left(h\left(s, u\left(s, \mathrm{u}_{0}^{\mathrm{k}}\right), \int_{0}^{a(s)} \mathrm{g}\left(\tau, u\left(\tau, \mathrm{u}_{0}^{\mathrm{k}}\right)\right) d \tau\right)-\right. \\
& \left.\frac{1}{\mathrm{~T}} \int_{0}^{T} h\left(s, u\left(s, \mathrm{u}_{0}^{\mathrm{k}}\right), \int_{0}^{a(s)} \mathrm{g}\left(\tau, u\left(\tau, \mathrm{u}_{0}^{\mathrm{k}}\right)\right) d \tau\right) d s\right) d s
\end{aligned}
$$

where $\mathrm{k}=1,2$, from (4.24), we get

$$
\begin{gathered}
\left.\left|\mathrm{u}\left(\mathrm{t}, \mathrm{u}_{0}^{1}\right)-\mathrm{u}\left(\mathrm{t}, u_{0}^{2}\right)\right| \leq \mid \mathrm{u}_{0}^{1}-u_{0}^{2}\right]+\frac{4(1-\alpha)}{(2-\alpha) N(a)}\left(k_{1}+a_{T} L_{1} k_{2}\right)\left|\mathrm{u}\left(\mathrm{t}, \mathrm{u}_{0}^{1}\right)-\mathrm{u}\left(\mathrm{t}, u_{0}^{2}\right)\right|+ \\
\frac{\alpha T}{(2-\alpha) N(\alpha)}\left(k_{1}+a_{T} L_{1} k_{2}\right)\left|\mathrm{u}\left(\mathrm{t}, \mathrm{u}_{0}^{1}\right)-\mathrm{u}\left(\mathrm{t}, u_{0}^{2}\right)\right|
\end{gathered}
$$

Therefore, we obtain that

$$
\begin{aligned}
\left|\mathrm{u}\left(\mathrm{t}, \mathrm{u}_{0}^{1}\right)-\mathrm{u}\left(\mathrm{t}, u_{0}^{2}\right)\right| & \leq\left|\mathrm{u}_{0}^{1}-u_{0}^{2}\right|+\left(2(1-\alpha)+\frac{\alpha T}{2}\right)\left(k_{1}+a_{T} L_{1} k_{2}\right)\left|\mathrm{u}\left(\mathrm{t}, \mathrm{u}_{0}^{1}\right)-\mathrm{u}\left(\mathrm{t}, u_{0}^{2}\right)\right| \\
& \leq\left|\mathrm{u}_{0}^{1}-u_{0}^{2}\right|+F_{1} F_{2}\left|\mathrm{u}\left(\mathrm{t}, \mathrm{u}_{0}^{1}\right)-\mathrm{u}\left(\mathrm{t}, u_{0}^{2}\right)\right|
\end{aligned}
$$

From equations (4.26), we have

$$
\left|\mathrm{u}\left(\mathrm{t}, \mathrm{u}_{0}^{1}\right)-\mathrm{u}\left(\mathrm{t}, u_{0}^{2}\right)\right| \leq F_{3}\left|\mathrm{u}_{0}^{1}-u_{0}^{2}\right|
$$

Substitutes (4.27) in (4.23), we get that (4.21)

Remark 1. [22]. Theorem 6 confirms the stability of the solution of the problem (1.1), when a slight change happens in the points $u_{0}$, then a slight change will happen in the function $\mu\left(0, u_{0}\right)$.

\subsection{Existence and uniqueness of periodic Solution of (1.1) with integral boundary condition}

In this section, we investigate the periodic solution of the problem (1.1) with integral boundary conditions:

$$
u(0)-u(T)=\int_{0}^{T} H(u(s)) d s
$$

Where the function $H(u(s))$ defined and continuous on are compact subset of $R$ and periodic on $t$ of periodic $\mathrm{T}$. 
Theorem 7. All assumptions of the Theorem 2 are satisfy, and the function $H(u(s))$ satisfies

$$
\left|H\left(u_{1}\right)-H\left(u_{2}\right)\right| \leq L_{2}\left|u_{1}-u_{2}\right|
$$

then the problem (1.1) and integral boundary condition(4.28) has unique solution if

$$
Q=\left(2(1-\alpha)+\frac{\alpha T}{2}\right)\left(k_{1}+a_{T} L_{1} k_{2}\right)+\frac{(1-\alpha+\alpha T)}{\alpha} L_{2}<1
$$

Proof. We define an operator $P: C[0, T] \rightarrow C[0, T]$

$$
\begin{aligned}
& P(u(t))=\mathrm{u}_{0}-\frac{(1-\alpha+\alpha t)}{\alpha T} \int_{0}^{T} H(u(s)) d t+ \\
& \frac{2(1-\alpha)}{(2-\alpha) N(a)}\left[h\left(t, u(t), \int_{0}^{a(t)} \mathrm{g}(\mathrm{s}, u(s)) d s\right)-\frac{1}{\mathrm{~T}} \int_{0}^{T} h\left(s, u(s), \int_{0}^{a(s)} \mathrm{g}(\tau, u(\tau)) d \tau\right) d s\right]+ \\
& \frac{2 \alpha}{(2-\alpha) N(\alpha)} \int_{0}^{t}\left(h\left(s, u(s), \int_{0}^{a(s)} \mathrm{g}(\tau, u(\tau)) d \tau\right)-\frac{1}{\mathrm{~T}} \int_{0}^{T} h\left(s, u(s), \int_{0}^{a(s)} \mathrm{g}(\tau, u(\tau)) d \tau\right) d s\right) d s
\end{aligned}
$$

Therefore, we get

$$
|P(u(t))-P(w(t))|=\left(\left(2(1-\alpha)+\frac{\alpha T}{2}\right)\left(k_{1}+a_{T} L_{1} k_{2}\right)+\frac{(1-\alpha+\alpha T)}{\alpha} L_{2}\right)|u(t)-w(t)|
$$

From (4.30), the operator $P$ satisfies contraction mapping, hence the problem (1.1) and (4.28) has unique solution.

Theorem 8. If the hypotheses and all the conditions of the theorem 2 and the inequality (4.29) are given, the following inequalities are satisfied:-

$$
\left|\sigma\left(0, \mathrm{u}_{0}\right)-\sigma_{\mathrm{m}}\left(0, \mathrm{u}_{0}\right)\right| \leq\left(k_{1}+a_{\Upsilon} L_{1} k_{2}+\frac{L_{1}}{\alpha}\right) Q^{\mathrm{m}}(1-Q)^{-1} M_{3}
$$

where

$$
\begin{aligned}
\sigma_{\mathrm{m}}\left(0, \mathrm{u}_{0}\right) & =\frac{1}{\mathrm{~T}} \int_{0}^{T} h\left(s, u_{m}(s), \int_{0}^{a(s)} \mathrm{g}\left(\tau, u_{m}(\tau)\right) d \tau\right) d s \\
& +\frac{(2-\alpha) \mathrm{N}(\alpha)}{2 \alpha \mathrm{T}} \int_{0}^{T} H\left(u_{m}(s)\right) d s
\end{aligned}
$$

holds for all $\mathrm{m} \geq 0$, here

$$
M_{3}=M_{1}+\frac{(1-\alpha+\alpha T)}{a} M_{2}
$$

and

$$
M_{2} \geq|H(u(t))|
$$

The proof of this theorem is direct. 
Theorem 9. Let the functions $h(s, u(s), z(t))$ and $H(u(t))$ be defined on the intervals $\left[c_{1}, d_{1}\right]$ on $R$ and periodic in $t$ of period $\mathrm{T}$, suppose that for all $\mathrm{m} \geq 0$, then the sequences of the functions $\sigma_{\mathrm{m}}\left(0, \mathrm{u}_{0}\right)$ which are defined in (4.32) satisfy the inequalities:-

$$
\left.\begin{array}{l}
\min _{u_{0} \in\left[c_{1}, d_{1}\right]} \sigma_{m}\left(0, u_{0}\right) \leq-\left(k_{1}+a_{T} L_{1} k_{2}+\frac{L_{2}}{\alpha}\right) Q^{m}(1-Q)^{-1} M_{3} \\
\max _{u_{0} \in\left[c_{1}, d_{1} 1\right.} \sigma_{m}\left(0, u_{0}\right) \geq\left(k_{1}+a_{T} L_{1} k_{2}+\frac{L_{2}}{\alpha}\right) Q^{m}(1-Q)^{-1} M_{3}
\end{array}\right\}
$$

Then the problem (1.1) with (4.28) has a periodic solution such that $u_{0} \in\left[c_{1}+M_{3}, d_{1}-M_{3}\right]$ where $M_{3}$ defined in (4.33).

This theorem's proof was similar to that of theorem 5 .

Theorem 10. Let the function $\sigma\left(0, \mathrm{u}_{0}\right)$ be defined by the equations (4.32), then the following inequalities yield:-

$$
\left|\sigma\left(0, \mathrm{u}_{0}\right)\right| \leq M+\frac{M_{2}}{\alpha}
$$

and

$$
\left|\sigma\left(0, \mathrm{u}_{0}^{1}\right)-\sigma\left(0, \mathrm{u}_{0}^{2}\right)\right| \leq E_{2} E_{3}\left|u_{0}^{1}-u_{0}^{2}\right|
$$

where

$$
E_{1}=2(1-\alpha)+\frac{\alpha T}{2}, \quad E_{2}=k_{1}+a_{T} L_{1} k_{2}+\frac{L_{1}}{\alpha}, \quad E_{3}=\left(1-\mathrm{E}_{1} E_{2}\right)^{-1}
$$

The proof of this theorem was similar to the proof of theorem 6 .

\section{Examples}

In this section contains two example to illustrate the previous theorems.

Example 5.1. Consider the following fractional integro-differential equation

$$
{ }_{0}^{C F} D_{t}^{0.7}(u(t))=\frac{1}{e^{t}+5} u(t)+\int_{0}^{t^{2}} \frac{1}{2(s+2)^{3}} \sin (u(s)) d s
$$

such that $t \in J=[0,2]$, with the initial condition $\mathrm{u}(0)=1$, where ${ }_{0}^{C F} D_{t}^{\alpha}$ denotes the fractional Caputo-Fabrizio derivative $(a=0.7 \in(0,1])$. Here $T=2, a(t)=t^{2}$,

$$
\begin{gathered}
h(t, u(t), z(t))=\frac{1}{e^{t}+5} u(t)+\int_{0}^{t^{2}} \frac{1}{2(s+2)^{3}} \sin (u(s)) d s \\
\mathrm{~g}(\mathrm{t}, u(t))=\frac{1}{2(s+2)^{3}} \sin (u(s))
\end{gathered}
$$

We obtain that $k_{1}=0.2, k_{2}=1, a_{T}=4, L_{1}=0.0625$,

so that $\Lambda=\left(2(1-\alpha)+\frac{\alpha r}{2}\right)\left(k_{1}+a_{T} L_{1} k_{2}\right)=0.585<1$.

Therefore, by Theorem 2 and Theorem 3, the fractional differential equation (5.1) has 
exactly one periodic solution.

Example 5.2. Consider the fractional integro-differential equation (5.1) with integral boundary conditions

$$
u(0)-u(1)=\int_{0}^{1} \frac{1}{2} \cos (u(t)) d t
$$

such that $t \in(0,1]$, where ${ }_{0}^{C F} D_{t}^{\alpha}$ denotes the fractional Caputo-Fabrizio derivative $(\alpha=$ $0.7 \in[0,1])$. Here $T=1,, a(t), h(t, u(t), \mathrm{z}(\mathrm{t})), \mathrm{g}(\mathrm{t}, u(t))$ are defined in previous example and

$$
H(u(t))=\frac{1}{2} \cos (u(t))
$$

we obtain that $k_{1}=0.2, k_{2}=1, a_{T}=1, L_{1}=0.0625$, and $L_{2}=0.5$, so that

$$
\mathrm{Q}=\left(2(1-\alpha)+\frac{a T}{2}\right)\left(k_{1}+a_{T} L_{1} k_{2}\right)+\frac{(1-\alpha+\alpha T)}{a} L_{2}=0.9637<1
$$

Therefore, by Theorem 7, the boundary value problem ( 5.1 ) and ( 5.2 ) has exactly one periodic solution.

\section{Conclusion}

In this paper,we studied the existence, uniqueness, and stability of periodic solutions of nonlinear fractional integro-differential equation (1.1) where ${ }_{0}^{C F} D_{t}^{\alpha}$ denotes the fractional Caputo-Fabrizio derivative with the initial condition, periodic boundary conditions, and integral boundary conditions by using technique successive approximations method and Banach fixed point theorem. Here conclude that we could investigate the existence, uniqueness, and stability of periodic solution of Caputo-Fabrizio fractional differential equation with integral boundary condition

$$
A u(0)-B u(T)=\sum_{i=1}^{m} C_{i} \int_{0}^{T} H_{i}(u(s)) d s,
$$

where $A, B$ and $C_{i}, i=1,2, \ldots, m$ are constants, and $H_{i}, i=1,2, \ldots, m$ are defined and continuous functions on $[0, T]$. Finally, some examples are presented to illustrate the previous theorems.

\section{References}

[1] M. Belmekki, J. J. Nieto, and R. R. Lopez. Existence of periodic solution for a nonlinear fractional differential equation. Boundary Value Problems, 2009:1-18, 2009.

[2] F. E. Bouzenna, M. T. Meftah, and M. Difallah. Application of the caputo-fabrizio derivative without singular kernel to fractional schrodinger equations. Pramana, 94, 2020 . 
[3] R. N. Butris and A. Sh. Rafeeq. Solutions for nonlinear system of fractional integro-differential equations with non-separated integral coupled boundary conditions. International Journal of Advanced Trends in Computer Science and Engineering, 9, 2020 .

[4] A. Cabada and T. Kisela. Existence of positive periodic solutions of some nonlinear fractional differential equations. Communications in Nonlinear Science and Numerical Simulation, 50:51-67, 2017.

[5] M. Caputo and Fabrizio. A new definition of fractional derivative without singular kernel. Progress in Fractional Differentiation and Applications, 2:73-85, 2015.

[6] M. Farkas. Periodic motions. Springer-Verlag, New York, 1994.

[7] M. Feckan and K. Marynets. Approximation approach to periodic bvp for mixed fractional differential systems. J. Comput. Appl. Math., 339:208-217, 2018.

[8] M. A. Imran, M. B. Riaz, N. A. Shah, and A. A. Zafar. Boundary layer flow of mhd generalized maxwell fluid over an exponentially accelerated infinite vertical surface with slip and newtonian heating at the boundary. Results in Physics, 8:1061-1067, 2018.

[9] K. Jothimani, K. Kaliraj, Z. Hammouch, and C. Ravichandran. New results on controllability in the framework of fractional integrodifferential equations with nondense domain. The European Physical Journal Plus, 134, 2019.

[10] A. A. Kilbas, H. M Srivastava, and J. J. Trujillo. Theory and Applications of Fractional Differential Equations. North-Holland Mathematics Studies, vol. 204. Elsevier, Amsterdam, 2006.

[11] V. Lakshmikantham, S. Leela, and V. Devi. Theory of Fractional Dynamic Systems. Cambridge Academic Publishers, Cambridge, 2009.

[12] J. Losada and J. J. Nieto. Properties of the new fractional derivative without singular kernel. Progress in Fractional Differentiation and Applications, 1:87-92, 2015.

[13] K. S. Miller and B. Ross. An Introduction to the Fractional Calculus and Fractional Differential Equations. Wiley, New York, 1993.

[14] Sh. A. Murad and S. B. Hadid. Existence and uniquness theorem for fractional differential equation with integral boundary condition. Journal of Fractional Calculus and Applications, , 3:1-9, 2012.

[15] Sh. A. Murad and A. Sh. Rafeeq. Existence of solutions of integro-fractional differential equation when $\alpha \in(2,3]$ through fixed point theorem. J. Math. Comput. Sci., 11:6392-6402, 2021. 
[16] Sh. A. Murad, H. J. Zekri, and S. Hadid. Existence and uniqueness theorem of fractional mixed volterra-fredholm integrodifferential equation with integral boundary conditions. International Journal of Differential Equations, 2011:1-16, 2011.

[17] A. Sh. Rafeeq. On the parametrization of nonlinear impulsive fractional integro-differential system with non-separated integral coupled boundary conditions. Science Journal of University of Zakho, 8:160-168, 2020.

[18] C. Ravichandran, K. Logeswari, and F. Jarad. New results on existence in the framework of atangana-baleanu derivative for fractional integro-differential equations. Chaos, Solitons and Fractals, 125:194-200, 2019.

[19] M. Ronto and A. M. Samoilenko. Numerical-Analytic Methods in the Theory of Boundary-Value Problems. World Scientific, Singapore, 2000.

[20] K. Salim and S. Abbas. Boundary value problem for implicit caputo-fabrizio fractional differential equations. International Journal of Difference Equations, 15:493-510, 2020.

[21] N. A. Shah, M. A. Imran, and F. Miraj. Exact solutions of time fractional free convection flows of viscous fluid over an isothermal vertical plate with caputo and caputo-fabrizio derivatives. Journal of Prime Research in Mathematics, 13:56-74, 2017.

[22] N. A. Sheikh, F. Ali, M. Saqib, I. Khan, and S. A. A. Jan. A comparative study of atangana-baleanu and caputo- fabrizio fractional derivatives to the convective flow of a generalized casson fluid. The European Physical Journal Plus, 132, 2017. 